\title{
I. Beitrag zur Kenntniss der Sulfate von Tierra amarilla bei Copiapó in Chile.
}

\author{
Von \\ G. Linck in Strassburg i. E. \\ (Mit Tafel I.) \\ (Mittheilungen aus dem mineralog. Institut der Universität Strassburg, \\ No. 11, Fortsetzung von Bd. 12, 424-452.)
}

\section{Verzeichniss der benutzten Literatur.}

Arzruni, A., Ueber den Coquimbit. Diese Zeitschr. 1879, 3, 516 u. f. (6).

B e r thi er, P., Analyse de l'alun de plume. Annal. d. min. 1820, 5, 259 u. f. (28).

Bertrand, E., De l'application du microscope à l'étude de la mineralogie. Bull. d. l. société minéralog. de France 1881, 4, 8 u. f. Ref, diese Zeitschr. 6, 294. (11).

B la as, J., Beiträge zur Kenntniss natürlicher wasserbaltiger Doppelsulfate. Sitzungsber. d. k. Akad. d. Wiss. Wien 1883, 87, 141 u. f. Diese Zeitschr. 10, 409. (26).

Derselbe, Ueber Römerit, Botryogen und natürlichen Magnesia-Eisenvitriol. Ebenda, 1884, 88, 1121 u. f. Diese Zeitschr. 10, 409. (31).

B reith a u t, A., Vollständiges Handbuch der Mineralogie. 1841. (5).

B r u n, A., Mineralogische Notizen. Diese Zeitschr. 1881, 5, 104 u. f. (19).

Dana, J. D., A system of mineralogy. 5. Aufl. 1873. (8).

D arwin, Ch., Geologische Beobachtungen über Süd-Amerika in den Jahren 1832-1836. Uebersetzt von Carus. 1878. (4).

Des Cloizeaux, M., Sur l'emploi des propriétés optiques biréfringantes pour la détermination des espèces cristallisées. Ann. d. min. 5 . sér. 1858, 14, 339 u. f. (7).

Derselbe, Notes sur les propriétés optiques de l'Érytrozincite, de la Raimondite et de la Copiapite. Bull. d. l. soc. minér. de France 1884, 4, 40 u. f. Diese Zeitschr. 6, 299. (12).

Domeyko, J., Mémoire sur la constitution géologique du Chili. Annal. d. mines. 4. sér. 1846, 9, 365 u. f. (3).

Derselbe, Mineralojia. 3. Aufl. Santiago 1879. (15).

Field, F., On some minerals from Chile. Quarterly journ. of the chemic. soc. of London $1862,14,153$ u. f. (20).

Groth, Zeitschrift f. Krystallogr. XV. 
Frenze1, A., Mineralogisches. Tschermak's mineralog. und petrograph. Mittheilungen 1888, 9, 397. (9).

G l ock er, Grundriss der Mineralogie. 1839. (27).

Grailich, J., Der Rómerit, ein neues Mineral aus dem Rammelsberge, Sitzber. d. k. Akad. d. Wissensch. Wien $1858,28,272$ u. f. (24).

Groth, P., Tabellarische Uebersicht der Mineralien. 2. Aufl. 1882. (14).

Haidinger, W., Handbuch der bestimmenden Mineralogie. 1845. (10).

H a us ma n n, J., Handbuch der Mineralogie. 1847. (18).

Lin ck, G. *), Ueber einige aus Chile stammende Sulfate. (Briefliche Mittheilung.) Neues Jahrbuch für Mineralogie etc. 1888, 1, 213. (32).

List, Analyse des Misy vom Rammelsberge bei Goslar. Annalen der Chemie und Phar macie $1830,73,239$ u. f. (25).

Meyen, F. J. F., Reise um die Erde in den Jabren 1830-1832. Berlin 1834. (1).

Pisani, F., Analyse de la Fibroferrite de Pallières. Comptes rend. de l'académie des sciences 59, 914 u. f. (23).

Prideaux, J., Notice of an undescribed native subsulfate of iron from Chile. Philosophical Magazine and Journ. of science 1841, 18. (17).

R a mmelsberg, Handbuch der Mineralchemie. 1. Aufl. 1860. (30).

Derselbe, - - $\quad$ - $\quad$ 2. Aufl. 1875. (13).

Rose, G., Ueber einige in Siid-Amerika vorkommende Eisenoxydsalze. Poggendorff's Annalen 1833, 27, 309 u. f. (2).

S mith, D., Reëxamination of American minerals. Part IV. The American journ. of sciences und arts 1854, 18. (21).

Tobler, E., Notiz über das Vorkommen von Kupfervitriol auf Stypticit aus Chile. Annalen der Chemie und Pharm. 1855, 94, 383 u. f. (22).

Ulrich, F., Ueber Misy aus dem Rammelsberge bei Goslar. Zeilschrift f. d. gesammten Naturwissenschaften. Halle 1854, 3, 22 u. f. (16).

Im nördlichen Chile, etwa unter dem 28. Grade südlicher Breite und dem 54. Grade westlich von Ferro liegt, nahe bei der Stadt Copiapó an dem gleichnamigen Flusse, der.Ort Bamillas. Nach Me yen ${ }^{*}$ ), welcher im Jahre 1831 Ramillas von Gopiapó aus besuchte, ist der Name zusammengezogen aus Tierra amarilla, eine Bezeichnung, welche die gelbe Farbe des dortigen Bodens andeuten soll.

Während das im Westen dieses Ortes anstehende Gestein von Meyen " als ein dunkel-lauchgrüner Serpentin, einen Uebergang in dichten Grúnstein bildend « bezeichnet wird, besteht in der Nähe von Ramillas ein mehr als 100-140 Fuss hoher Bergabhang ausschliesslich aus Eisensulfaten. Die Sulfate sind oberflächlich zu einer gelben Erde verwittert und haben auch der Luft einen bedeutenden Gehalt an schwefliger Säure und Schwefelsäure mitgetheilt.

*) Die in dieser vorläufigen Mittheilung angegebene Zusammensetzung der Sulfate musste bei der Ausarbeitung theilweise modificirt werden; auch stellte sich heraus, dass das dort als Bückingit bezeichnete Mineral jedenfals als identisch mit dem Römerit zu betrachten ist.

**) l. c. (1) $392 \mathrm{ff}$. 
Die Mächtigkeit der » Krystalllager « schätzte Me ye n auf elwa 15 Fuss. Schon zur Zeit seines Besuches waren sie von kleinen Stollen förmlich durchwưhlt, weil die Bewohner jener Gegend Gold und Edelsteine darunter verborgen glaubten.

Me yen brachte von seiner Reise Stufen jener Salze mit und G. Ros e*), welcher dieselben untersuchte, fand folgende fünf verschiedene Sulfate:

1. Neutrales schwefelsaures Eisenoxyd mit Krystallisationswasser. $\mathrm{Fe}_{2} \mathrm{~S}_{3} \mathrm{O}_{12}+9 \mathrm{H}_{2} \mathrm{O}$ (Coquimbit).

2. Erstes basisch schwefelsaures Eisenoxyd mit Krystallisationswasser. $\mathrm{Fe}_{2} \mathrm{~S}_{5} \mathrm{O}_{20}+18 \mathrm{H}_{2} \mathrm{O}$ (Copiapit).

3. Zweites basisch schwefelsaures Eisenoxyd mit Krystallisalionswasser. $\mathrm{Fe}_{4} \mathrm{~S}_{4} \mathrm{O}_{16}+\mathrm{g}_{1} \mathrm{H}_{2} \mathrm{O}$ (Stypticit).

4. Neutrale schwefelsaure Thonerde mit Krystallisationswasser. $\mathrm{Al}_{2} \mathrm{~S}_{3} \mathrm{O}_{12}+18 \mathrm{H}_{2} \mathrm{O}$ (Haarsalz).

5. Schwefelsaures Kupferoxyd.

Eine ausfuhrliche Beschreibung des Gebietes von Copiapó lieferte im Jahre 1846 J. Domeyko**). Ich entnehme derselben, dass in jener Gegend Gold-, Silber- und Kupfer-führende Gänge schon seit alter Zeit abgebaut werden. Die Erzgänge führen, wo sie taub werden, fast ausschliesslich Schwefelkies. Sie setzen in Sedimentgesteinen auf, welche Granit uberlagern und aus compacten Schiefern, thonigen Mergeln, Gypsen und Porphyrtuffen (porphyres argileux) bestehen, im Allgemeinen nach Osten einfallen und eine Mächtigkeit von etwa 300 Metern erreichen. Diese Gesteine durchsetzt auch ein Gang von Eisenkies, welcher viele Eisensulfate enthält und über den sich Domeyko wie folgt äussert: "Tout près du fond de la vallée et sur l'escarpement de la rive gauche, en face de la ferme de Tierra amarilla, affleure un filon piriteux, contenant beaucoup de sulfate de fer, de cuivre et d'alumine. "

Die Auffassung von Domeyko entspricht im Wesentlichen auch der von $\mathrm{D}$ a rwi ( $^{* *}$ ) aus den Jahren 1832 -1836; nur lässt Dar wi n die Frage offen; ob die Sulfate einen Gang oder ein Lager bilden und ob die unter dem Gyps liegenden Gesteine nicht umgewandelte Thonschiefer sind.

Auf einer Reise durch Chile hat im Jahre 1883 auch G. St e in mann den Ort Tier ra marilla besucht und dem mineralogischen Institut der Universität Strassburg eine grössere Zahl von Stufen milgebracht, welche mir zur Untersuchung anvertraut wurden.

Herr Prof. Stein mann theilt mir uber die von ihm gemachten Funde

*) 1. c. (2).

**) 1. c. (3) $377-379$.

***) l. c. (4) $326-327$. 
Folgendes mit: „Etwa $15 \mathrm{~km}$ oberhalb der Stadt Go piapó liegt die Eisenbahnstalion Tierra a marilla (490 $\mathrm{m}$ uber dem Meere), wo sich die bedeutenste Kupferschmelze der Umgegend befindet. Schräg gegenúber und etwas unterhalb des Ortes liegt auf der linken Thalseite nur wenig höher als die Thalsohle die Mina Alcaparrosa. Das ausgedehnte Vorkommen von Eisensulfaten im Ausgehenden des Ganges ist fur die Namengebung *) des Bergwerkes und des Ortes Tierra amarilla massgebend gewesen. Der Gang ist weniger durch hohen Gehalt an Kupfer, als durch seine mehrere Meter betragende Mächtigkeit ausgezeichnet. «

„Wir verdanken J. Dome y k $0^{*}$ **) eine Beschreibung der Alcaparrosa aus dem Jahre 1846, welcher ich wenig Neues hinzuzufugen habe, da mein Besuch nur ein sehr fluchtiger war. «

»Das Gestein, in welchem der Gang der Alcaparrosa aufsetzt, scheint ein silificirter Porphyrtuff zu sein, wie solche auf der linken Thalseite des $R$ io de Copiapó in der Năhe von Tierra a marilla in recht bedeutender Ausdehnung entwickelt sind. «

„Da das Gestein in der Nähe des Erzganges durch Einwirkung von Schwefelsäure stark verändert worden ist, so ist eine genaue Bestimmung sebr erschwert. Darwi n $^{* *}$ ) erklärte es für umgewandelten Thonschiefer, während Dome yko in demselben, wohl mit Recht, ein Glied der "Porphyrformation " vermuthete. Thatsache ist, dass das Liegende des Gesteinscomplexes in jener Gegend gegen Osten von Hornblendegranit, das Hangende von Porphyrtuffen resp. -Decken mit darüber folgendem Neocomkalk gebildet wird. Hierdurch ist das Alter als präcretacisch bestimmt und kann nach analogen Lagerungsverhältnissen in der Umgegend als jurassisch angesehen werden. «

"Der mehrere Meter breite Erzgang führt, soweit ich ermitteln konnte, ausschliesslich compacten Schwefelkies mit einem geringen Kupfergehalte. Erst in jungerer Zeit, nachdem die Kiese durch die erleichterten Communicationen besseren Absatz gefunden haben, ist man zur Förderung des Erzes selbst geschritten. In fruherer Zeit beruhte der Ruf des Bergwerkes ausschliesslich auf dem Reichthum des Ausgehenden an Sulfaten, die man als Magistral benutzte, d. h. um die geschwefelten Silbererze zur Amalgamation geeignet zu machen. Das bis zu einer Tiefe von etwa $7 \mathrm{~m}$ aus Sulfaten gebildete Ausgehende des Ganges ist jetzt fast vollständig abgebaut. Am Eingange

*) Caparrós oder Alcaparros ist die spanische Bezeichnung lür Vitriol und die gelbe Farbe der Zersetzungsproducte der Sulfate hat die Bezeichnung gelbe "Erde“ (Tierra amarilla) hervorgerufen. - Caparrós, ein offenbar maurisches Wort, diurfte mit Caparrón (= Knospe, Trieb) zusammenhängen und in ähnlicher Weise wie unser deutsches Wort Ausblühung zu deuten sein.

**) 1. c. (3).

***) l. c. (4). 
zum Bergwerke trifft man kleinere Butzen der Sulfate, namentlich in der Nähe des umgebenden Gesteines zierliche Efflorescenzen von Haarsalz. Im Uebrigen war man für Aufsammlungen auf eine Tonne, welche etwa zur Hälfte mit kopfgrossen Stưcken der Sulfate gefült war, und auf die Liebenswürdigkeit meines Fuhrers, des damaligen Hüttendirectors von Tierra a marilla, Herrn Geisse angewiesen, welcher mir eine freie Auswahl unter den Stucken seiner Sammlung gestattete. "

Die Untersuchung der von Herrn Prof. Steinmann mitgebrachten Salze hat mehrere neue Sulfate ergeben, deren Beschreibung ich im Folgenden mittheilen will.

\section{Coquimbit (Breithaupt).}

Der Coquimbit, von Breithaupt*) so benannt nach der Stadt Coquimbo in Ghile, kam zuerst durch Meyen von dem Fundorte Ramillas (Tierra amarilla) nach Europa und wurde von G. Rose * $^{*}$ ) untersucht. Rose fand, dass dem Mineral die Zusammensetzung $\mathrm{Fe}_{2}\left(\mathrm{SO}_{4}\right)_{3}+$ $9 \mathrm{H}_{2} \mathrm{O}$ zukomme, dass dasselbe hexagonal krystallisire, und dass die Krystalle eine Combination der Flächen $\{10 \bar{T} 0\} \infty P$, $\{10 \overline{1} 1\} P$ und $\{0001\} 0 P$ darstellen. Für das Axenverhältniss fand er $a: c=1: 1,562$, und ausserdem bemerkte er eine unvollkommene Spaltbarkeit nach Prisma und Pyramide.

Eine Angabe über das specifische Gewicht findet sich zuerst bei B r ei thaupt, welcher dasselbe zu "2,092 wahrscheinlich ein wenig höher « angiebt.

Eine Zusammenstellung des uber den Coquimbit Bekannten und eine Anzahl neuer Untersuchungen verdanken wir Arzruni***). Derselbe unterzog im Jahre 1879 die Rose'schen Originalstücke einer erneuten Bearbeitung. Er bestätigte die Resultate Rose's und auch den von Des Gloizeauxt) erkannten positiven Gharakter der Doppelbrechung. Ausserdem fand er noch folgende Formen an den Krystallen: $\{1 / \overline{2} 0\} \infty P 2$, $\{1 / \overline{2} 2\} P 2,\left\{10 T_{2}\right\}_{2} P,\{10 T 3\} \frac{1}{3} P$ und $\{1 / \overline{2} 1\} 2 P 2$. Auch bestimmte er die Brechungsexponenten

$$
\begin{aligned}
\text { für Na-Licht } \omega=1,5455, & \varepsilon=1,5547 \text { und } \\
\text { - Li-Licht } \omega=1,5376, & \varepsilon=1,5468
\end{aligned}
$$

und damil war im Gegensalze zu der Angabe von D e s Cl oi zeaux erkannt, dass die Doppelbrechung keine starke sei.

Die grossen Stufen, welche mir zur Verfügung standen (im Ganzen

\footnotetext{
*) 1. c. (5) 2, 100 .

**) 1. c. (2) 310 .

***; 1. c. (6).

†) 1. c. (7) 347 .
} 
etwa 40 Pfund), haben mir ein vortreffliches Material für die krystallographische Untersuchung und die Analyse geliefert.

Die Farbe des Coquimbit ist meist lichtbläulichviolett, seltener grünlich oder bei ganz kleinen Krystallen äusserst wenig gelblich oder bläulich.

Die kleinen Krystalle eignen. sich am besten zum Messen. Die Untersuchung derselben hat ergeben, dass der Coquimbit nicht hexagonalholoëdrisch, sondern in der rhomboëdrischen Hemiëdrie krystallisirt. Die Mehrzahl der Krystalle zeigt allerdings eine holoëdrische Ausbildung. Sie entsprechen der Combination $c=x\{0001\} 0 R, m=x\{10 \bar{T} 0\} \infty R$, $r=x\{10 T 1\}+R, r^{\prime}=x\{01 T 1\}-R$ (Taf. I, Fig. 1) und zwar sind meist Basis und Prisma vorherrschend, seltener tritt die Basis gegenüber den anderen Flächen zuruck.

Neben den eben beschriebenen Krystallen finden sich aber auch in reichlicher Anzabl solche von ausgesprochen rhomboëdrischem Habitus. Auch sie gebören zwei verschiedenen Typen an. Die einen zeigen vorherrschend $c=x\{0001\} 0 R$ und $o=x\{30 \overline{3} 2\}+\frac{3}{2} R$, untergeordnet $p=$ $x\{11 \overline{2} 0\} \infty P 2$ und $r=x\{10 \bar{T} 1\}+R$ (Fig. 2). Bei nur flüchtiger Betrachtung gleichen diese Krystalle einer Combination des regulären Oktaëders mit dem Rhombendodekaëder; auch die Winkel weichen nur sehr wenig von denjenigen der regulären Combination ab.

Der andere Typus ist mehr prismatisch ausgebildet und flächenreicher (Fig. 3). Gross entwickelt sind daran: $c=x\{0001\} 0 R, m=x\{10 \bar{T} 0\} \infty R$, $o=x\{30 \overline{3} 2\}+\frac{3}{2} R$.

Klein ausgebildet; $p=x\{11 \overline{2} 0\} \infty P 2, a=x\{30 \overline{3} 1\}+3 R, q=$ $x\{30 \overline{3} 3\}+\frac{3}{5} R, a^{\prime}=x\{03 \overline{3} 1\}-3 R, r^{\prime}=x\{01 \overline{1} 1\}-R, b=x\{03 \overline{3} 4\}-\frac{3}{4} R$, $n=\dot{x}\{03 \overline{3} 7\}-\frac{3}{7} R$.

Die Flächen $n, b$ und $a$ fehlen ziemlich häufig oder sind nur als sehr schmale Abstumpfungen der entsprechenden Kanten vorhanden.

Ist schon die Art der Ausbildung dieser Krystalle ein Beweis für die Zugehörigkeit des Coqu i mbitt zur rhomboëdrischen Abtheilung des hexagonalen Systemes, so liefert eine vollkommene Bestătigung dieser Behauptung die Beobachtung von Zwillingen. Dieselben besitzen die Ausbildung wie in Fig. 4.

Einzelne Individuen besitzen vorherrschend die Flächen $c=x\{0001\} 0 R$, $0=x\{30 \overline{3} 2\}+\frac{3}{2} R, p=x\{11 \overline{2} 0\} \infty P 2, a^{\prime}=x\{03 \overline{3} 1\}-3 R$ und mehr zurück tretend noch $b=x\{03 \overline{3} 4\}-\frac{3}{4} R$ und $r=x\{10 \overline{1} 1\}+R$. Zwillingsebene ist $0 R$ und nach dieser Fläche sind die Krystalle auch verwachsen, so nämlich, dass die Flächen $\infty P 2$ bei beiden Individuen in eine Ebene fallen und keine einspringenden Winkel entstehen. Zuweilen sind auch zwei Individuen von dem Typus der Fig. 2 in umgekehrter Stellung mit einander verwachsen. Die $\mathrm{Zwillingsgrenze}$ wird manchmal noch besonders deutlich 
durch kleine, schwarze, entlang derselben eingelagerte Erzpartikel und durch eine bei einem Theile der Krystalle auf $\infty P 2$ leicht sichtbare Streifung, welche der Combinationskante mit $x\{30 \overline{3} 2\}+\frac{3}{2} R$ parallel verläuft. Bei denjenigen Krystallen, welche die Streifung nicht zeigen, kann dieselbe durch kurz andauerndes Aetzen mit sehr verdünnter Salzsäure hergestellt werden.

Im Nachstehenden sind die wichtigsten der gemessenen und berechneten Winkel zusammengestellt.

\begin{tabular}{|c|c|c|}
\hline & Gemessen : & Berechnet \\
\hline$m: r^{\prime}=(01 \bar{T} 0):(01 \bar{T} 1)$ & $={ }^{*} 29^{\circ} \quad \prime^{\prime}$ & - \\
\hline$m: m=(10 \mathrm{~T} 0):(01 \overline{1} 0)$ & $=601$ & $60^{\circ} 0^{\prime}$ \\
\hline$m: p=-(10 \pi 0):(11 \overline{2} 0)$ & $=30$ & 30 \\
\hline$m: o=(10 \bar{T} 0):(30 \overline{3} 2)$ & $=2032$ & 2018 \\
\hline$m: q=(10 \overline{1} 0):(30 \overline{3} 5)$ & $=4228$ & 4245 \\
\hline$m: a=(10 \overline{1} 0):(30 \overline{3} 1)$ & $=1032$ & 1029 \\
\hline$m: b=(01 \bar{T} 0):(03 \overline{3} 4)$ & $=36 \cdot 30$ & 3629 \\
\hline$m: n=(0170):(03 \overline{3} 7)$ & $=5214$ & $52 \quad 19$ \\
\hline$o: b=(30 \overline{3} 2) ;(03 \overline{3} 4)$ & $=54$ & $54 \quad 19$ \\
\hline$o: p=(30 \overline{3} 2):(11 \overline{2} 0)$ & $=36$ & $35 \quad 41$ \\
\hline
\end{tabular}

Für die Zwillinge :

$$
\begin{aligned}
& 0: \underline{o}=(30 \overline{3} 2):(30 \overline{3} \overline{2})=4049^{\prime} \quad 4036 \\
& a^{\prime}: \underline{a}^{\prime}=(03 \overline{3} 1):(03 \overline{3} \widehat{T})=2047 \quad 2058
\end{aligned}
$$

Aus dem Winkel $m: r^{\prime}=(01 T 0):(01 T 1)$ berechnet sich das Axenverhältniss :

$$
\begin{aligned}
a: c= & 1: 1,5613 \\
& (1: 1,562 \text { Rose }) \\
& (1: 1,5645 \text { Arzruni), }
\end{aligned}
$$

ein Verhältniss, welches von dem von G. Ro se angegebenen nur wenig abweicht.

Die Krystalle erreichen eine Grösse bis zu 1,5 cm nach allen drei Richtungen. Die grösseren Individuen sind aber meist durch andere Salze stark verunreinigt. Die Flächen insbesondere der kleinen Kryställchen sind sehr glatt und glänzend. Trotzdem ergaben die an verschiedenen Krystallen angestellten Messungen stets um mehrere Minuten von einander abweichende' Werthe, aus welchen daher das Mittel genommen wurde.

Die optische Untersuchung bestätigte das bisher uber den Coquimbit Bekannte. Dünne und ganz besonders reine, einschlussfreie Platten und Krystalle zeigen im convergenten Lichte das ungestörte Interferenzbild einaxiger Mineralien. Die dickeren Individuen hingegen und diejenigen, welche reich an Einschlissen sind, sind durchweg zweiaxig 'mit einem 
Axenwinkel bis zu etwa $18^{0}$ für $\mathrm{Na}$-Licht in Luft. Der Axenwinkel wechselt sowohl in Beziehung auf Lage als auf Grösse in demselhen Individuum vielfach und in ganz unregelmässiger Weise.

Zur Bestimmung des Brechungsexponenten wurden drei Prismen geschliffen. Eines derselben war besonders gut gelungen. Eine Fläche war natürliche Prismenfläche und die andere lag genau in der Prismenzone. Die Messung ergab nachstehende Werthe, denen ich die von Arzruni*) erhaltenen in Klammern beifüge :

\begin{tabular}{|c|c|c|c|c|}
\hline \multirow{3}{*}{$N a-$ Licht } & $\vartheta$ & $\vartheta^{\prime}$ & $\omega$ & $\varepsilon$ \\
\hline & $18^{0} 32^{\prime}$ & $18^{0} 43 \frac{1}{2}^{\prime}$ & 1,5519 & 1,5575 \\
\hline & $1\left(17 \quad 14 \frac{1}{2}\right.$ & $1732 \frac{1}{2}$ & 1,5455 & 1,5547) \\
\hline \multirow{2}{*}{$L i$-Licht } & $\int \begin{array}{ll}18 & 19 \frac{1}{2}\end{array}$ & $1829 \frac{1}{2}$ & 1,5469 & 1,5508 \\
\hline & $1(1659$ & $1717^{4}$ & 1,5376 & $1,5468\}$ \\
\hline
\end{tabular}

Hierbei bedeutet $\vartheta$ und $\vartheta^{\prime}$ den Ablenkungswinkel für $\omega$ resp. $\varepsilon$ und $\varphi$ den Prismenwinkel.

Die gefundenen Grössen weichen also sehr wenig von denen Arzru$n \mathrm{i}$ 's ab, nur kann ich die Farbendispersion ebensowenig eine beträchtliche nennen wie die Doppelbrechung.

Behandelt man einen Krystall von Coquimbit kurze Zeit mit erwärmtem Wasser, dem eine geringe Quantität Salzsäure zugesetzt ist, so entstehen Aetzfiguren, wie sie der rhomboëdrischen Hemiëdrie des Sulfates entsprechen. Am leichtesten und schönsten bilden sie sich auf der Basis $0 R$ als gleichseitige Dreiecke, welche in ihrer Stellung einem negativen Rhomboëder entsprechen.

Weniger deutlich sind die Aetzfiguren auf den Rhomboëderflächen.

Auf den positiven Rhomboëderflächen habe ich theils gleichschenklige Dreiecke beobachtet, welche an der Spitze durch eine Gerade abgestumpft sind und deren Winkel an der Spitze etwa $30^{\circ}-35^{\circ}$ betragen würde; theils entstanden Figuren, welche einem gleichschenkligen, unten durch eine flache bogenförmige Linie geschlossenen Dreiecke ebenfalls mit einem Winkel von $20^{\circ}-30^{\circ}$ an der Spitze gleichen. Die ersteren kehren ihre Spitze nach oben, die letzteren nach unten.

Auf den negativen Rhomboëdern habe ich nur sehr selten Aetzfiguren beobachten können, sie gleichen denen der positiven Rhomboëderflächen und kehren ihre Spitze nach oben.

Es gelang nicht, auf den steilen Rhomboëdern oder auf $\infty R$ Aetzfiguren zu beobachten. Hingegen bemerkt man auf $\infty P 2$ nach dem Aetzen meist

*) 1. c. (6), 522 . 
eine diagonale Streifung, welche der Combinationskante dieser Fläche mit dem positiven Rbomboëder $x\{30 \overline{3} 2\}+\frac{3}{2} h$ parallel geht.

Spaltbarkeit ist beim Coquimbit, wie schon G. Ros e gefunden hat, und ich nur bestätigen kann, nach drei Richtungen vorhanden, und zwar nach $\infty R$, nach $+R$ und nach $-R$. Sie ist nach allen drei Richtungen gleichwertbig und unvollkommen.

Die Härte des Minerals ist 2-2 $\frac{1}{2}$.

Die Bestimmung des specifischen Gewicbts wurde mittelst Thoule t scher Lösung und der Westphal'schen Wage ausgefuhrt, da diese Art der Bestimmung ausserordentlich viel einfacher ist und gegenutber der Ermittelung mit dem Pyknometer den Vortheil voraus bat, dass jegliche Correction der directen Beobachtung überflüssig wird. Als Schwierigkeil stand der Bestimmung nur der Umstand der Löslichkeit der Substanz in Wasser gegenüber. Um die Einwirkung der Flüssigkeit auf den Coquimbit zu vermeiden oder wenigstens möglichst zu vermindern, wurden einige ca. 20-30 cmm grosse vollkommen wasserklare Krystalle mit einer ganz dünnen Schicht Paraffin uberzogen und zwar in der Weise, dass eine Lösung von ganz wenig Paraffin in viel Aether hergestellt und davon einige Tropfen uber die Krystalle weggegossen wurden. An so behandelten Krystallen fand ich das spec. Gew. schwankend zwischen 2,079 und 2,114. Es könnte dieser Art der Bestimmung der Vorwurf gemacht werden, dass das specifische Gewicht durch die die Krystalle umgebende Paraffinschicht wesentlich beeinflusst würde. Dies ist jedoch nur in sehr geringem Maasse der Fall, wie ein Versuch, der mil ganz kleinen Spaltungsstuckchen (ca. 6-8 cbmm gross) von isländischem Doppelspath angestellt wurde, lehrte. Die Rhomboëderchen zeigten zwischen dem specifischen Gewicht vor dem Ueberziehen mit Paraffin und dem nach dieser Operation nur eine Differenz von $0,004-0,006$.

Es durfte also das Mittel aus den verschiedenen Beobachtungen, welche ich an Coquimbitkrystallen, die nach der eben beschriebenen Methode behandelt waren, gemacht habe, der Wirklichkeit sehr nahe kommen. Somit finde ich das specifische Gewicht des $\mathrm{Go}$ qu imbit bei $15^{0} \mathrm{C}$. ziemlich ubereinstimmend mit Breit ha pt*) (2,092) gleich 2,105.

Ueber die chemische Zusammensetzung des Goquimbit sind wir schon seit der Untersuchung Ros e's**) vollständig im Klaren und es war somit zu erwarten, dass eine erneute mit völlig reinem Material ausgefuhrte Analyse die Resultate jerres ausgezeichneten Forschers nur bestätigen würde, wie es auch die Analysen Bamberger's***) gethan haben.

*) 1. c. (5), $2,100$.

**) l. c. (2) 311 .

***) I. c. (6) 523 . 
G. Rose fand, dass das Mineral neutrales schwefelsaures Eisenoxyd mit neun Molekülen Krystallwasser sei, also der Formel $\mathrm{Fe}_{2} \mathrm{~S}_{3} \mathrm{O}_{12}+9 \mathrm{H}_{2} \mathrm{O}$ entspreche.

Auch die von mir ausgeführte Analyse simmt mit dieser Annahme vollständig überein. Zur besseren Uebersicht ist sie unten mit denjenigen von G. Rose*) und Bamberge $\mathrm{r}^{* *}$ ) zusammengestellt.

\begin{tabular}{ccccc} 
& Rose: & \multicolumn{2}{c}{ Bàmberger: } & Linck: \\
$\mathrm{SiO}_{2}$ & 0,31 & 1,17 & 0,94 & $1,29 * * *)$ \\
$\mathrm{SO}_{3}$ & 43,55 & 41,68 & 43,10 & 41,48 \\
$\mathrm{Fe}_{2} \mathrm{O}_{3}$ & 24,11 & 22,63 & 22,16 & 27,86 \\
$\mathrm{Al}_{2} \mathrm{O}_{3}$ & 0,92 & 4,70 & 4,78 & Spur \\
$\mathrm{CaO}$ & 0,73 & - & - & - \\
$\mathrm{MgO}$ & 0,32 & 0,46 & 0,19 & Spur \\
$\mathrm{H}_{2} \mathrm{O}$ & 30,10 & 28,94 & 28,83 & 28,77 \\
\hline Summe & 100,04 & 99,58 & 100,00 & 99,40
\end{tabular}

Berechnet man diese Analysen nack Abzug der Magnesia als Bittersalz und des Kalkes als Gyps auf 100, so erhält man:

\begin{tabular}{|c|c|c|c|c|}
\hline \multirow[b]{2}{*}{$\mathrm{SO}_{3}$} & \multirow{2}{*}{$\begin{array}{l}\text { Rose: } \\
43,58\end{array}$} & \multicolumn{2}{|c|}{ Bamberger: } & \multirow{2}{*}{$\begin{array}{r}\text { Linck } \\
42,28\end{array}$} \\
\hline & & 42,64 & 43,64 & \\
\hline $\mathrm{Fe}_{2} \mathrm{O}_{3}$ & 25,10 & 23,68 & 22,64 & 28,40 \\
\hline $\mathrm{Al}_{2} \mathrm{O}_{3}$ & 0,96 & 4,92 & 4,88 & - \\
\hline $\mathrm{H}_{2} \mathrm{O}$ & 30,36 & 28,76 & 28,84 & 29,32 \\
\hline ame & 00,00 & 100,00 & 100,00 & 100,00 \\
\hline
\end{tabular}

Die Formel $\mathrm{Fe}_{2} \mathrm{~S}_{3} \mathrm{O}_{12}+9 \mathrm{H}_{2} \mathrm{O}$ verlangt in 100 Theilen:

$\begin{array}{ll}\mathrm{SO}_{3} & 42,70 \\ \mathrm{Fe}_{2} \mathrm{O}_{3} & 28,47 \\ \mathrm{H}_{2} \mathrm{O} & 28,83\end{array}$

Es findet also eine vorzügliche Uebereinstimmung der Analysen mit der von G. Ros e aufgestellten Formel statt.

Was die Löslichkeitsverhältnisse des Coquimbit anlangt, ist zu erwähnen, dass derselbe in Wasser zwar langsam, aber vollkommen löslich ist. Die Lösung reagirt sauer, schmeckt herb, adstringirend und trübt sich beim Kochen unter Abscheidung basischer Sulfate.

Beim Liegen an der Luft, besonders an trockener Luft, erfährt der Co-

*) I. c. (2) 311 .

**) 1. c. (6) 523 .

***) Als Quarzsand den Kryställchen anhängend.

$\therefore$ ) Lus der Diflerenz. 
quimbit eine Veränderung. Er bedeckt sich zunächst, offenbar in Folge von Wasserverlust, mil einem weissen Pulver. Erst beim Einwirken von Atmosphärilien findet eine Abnahme des Schwefelsäuregehaltes statt.

Der Coqui mbit ist bis jetzt nur von zwei Localitäten bekannt. Der eine Fundort, welcher wohl in europäischen Sammlungen allein vertreten ist und gewöbnlich kurzweg als $\mathrm{Copiapó} \mathrm{oder} \mathrm{Ghile} \mathrm{bezeichnet} \mathrm{wird,}$ ist Tierra amarilla. Ausserdem giebt $\mathrm{Dana*}$ ) als weiteren Fundort Cal ama in Bolivien an, wo er den grösseren Theil eines bedeutenden Hügels bilden soll. Der letztere Fundorl ist wahrscheinlich mit dem von Hohmann**) angegebenen Punkte (N. v. Sierra Garda bei Caracoles) identisch. Gehört der Blakeït Dana's***), wie ich zwar vermuthe + ), aber wegen Unzugänglichkeit der Originalabhandlung nicht mit Bestimmtbeit behaupten kann, auch hierher, so ist Coquimbo als dritter Fundort zu nennen. Dass andere Vorkommnisse, wie die vom R m melsberg bei Goslar, vom Vesuv und von der Algodonbay in Bolivien nicht zum Coquimbit zu rechnen sind, hat schon Arzrunitt) mit genügender Schärfe hervorgehoben.

\section{Quenstedtit (Linck).}

Neben dem Coquimbit finden sich in einigen Stufen röthlichviolette, tafelförmige, gypsähnliche Krystalle, deren Dimensionen bis $5 \mathrm{~mm}$ in der Länge, $1-2 \mathrm{~mm}$ in der Breite und $1 \mathrm{~mm}$ in der Dicke betragen. Sie sind vollkommen durchsichtig. Die angestellten krystallographischen Untersuchungen ergaben folgendes Resultat.

Krystallsystem : Monosymmetrisch.

$$
\begin{gathered}
a: b: c=0,3942: 1: 0,4060 \\
\beta=77^{0} 58^{\prime} .
\end{gathered}
$$

Die beobachteten Formen sind: $b=\{010\} \infty R_{\infty}, m=\{110\} \infty P$, $p=\{350\} \infty R_{\frac{5}{3}}, q=\{011\} R \infty, r=\{0.11 .10\} \frac{11}{10} R \infty, s=\{085\} \frac{8}{5} R \infty$, $t=\{074\} \frac{7}{4} R \infty, u=\{0.15 .8\} \frac{15}{8} R \infty, v=\{094\} \frac{9}{4} R \infty, w=\{052\} \frac{5}{2} R \infty$ (Fig. 5).

Die meisten Krystalle sind verlängert nach der Klinodiagonale und

*) l. c. (8) 651 .

**) 1. c. (9) 399 s. bei Frenzel.

***) J. c. (8) 652 bei Volta it.

4) Eine Verwechslung der rhomboëdrischen Coquimbilkryslalle, Combination $0 R,+\frac{3}{2} R$, mit dem regulären Oktaëder ist einerseits wegen der Aehnlichkeit der Winkel $(0001):(30 \overline{3} 2)=69042^{\prime}$ und $(30 \overline{3} 2):(03 \overline{3} 2)=71022^{\prime}$ und der Oktaëderwinkel $\left.=70^{\circ} 32^{\prime}\right)$ und andererseits wegen der schwachen Doppelbrechung des Coquimbit sehr leicht möglich.

tt) l. c. (6) 519. 
zeigen eine sehr flächenreiche Klinodomenzone, in welcher jedoch meist die eine oder die andere oder mehrere der oben bezeichneten Formen feblen. Die Klinodomenflächen sind häufig längsgestreift und daher die Reflexe nicht immer befriedigend. Die Krystalle von der einfachen Combination $b=\{010\} \infty R \infty, m=\{110\} \infty P, w=\{052\} \frac{5}{2} R \infty$ (Fig. 6) sind sellener.

Die gemessenen und berechneten Winkel sind folgende:

\begin{tabular}{|c|c|c|}
\hline & Gemessen : & Berechnet: \\
\hline$b: m=(010):(110)$ & $={ }^{*} 68^{0} 55^{\prime}$ & - \\
\hline$b: t=(010):(074)$ & $=* 3512$ & - \\
\hline$q: m=(011):(T 10)$ & $=* 8723$ & - \\
\hline$b: w=(010):(052)$ & $=4532$ & $45042^{\prime}$ \\
\hline$b: v=(040):(094)$ & $=4815$ & $48 \quad 13$ \\
\hline$b: u=(010):(0.15 .8)$ & $=5324$ & 5321 \\
\hline$b: s=(010):(085)$ & $=5722$ & 5723 \\
\hline$b: r=(010):(0.11 .10)$ & $=6639$ & $66 \quad 15$ \\
\hline$b: q=(010):(011)$ & $=6835$ & $68 \quad 12$ \\
\hline$m: t=(110):(074)$ & $=6914$ & 6834 \\
\hline$b: p=(010):(350)$ & $=5745$ & 5717 \\
\hline
\end{tabular}

Ausserdem wurde auf $b=\{010\} \infty R \infty$ der , von der Verticalaxe und der Klinodiagonale gebildete Winkel $(\beta)$ zu ungefähr $78^{\circ}$ bestimmt.

Was die optischen Eigenschaften des Minerals anlangt, konnte nur festgestellt werden, dass die optische Axenebene im Klinopinakoid liegt und zwar so, dass die erste Mittellinie im stumpfen Winkel $\beta$ gegen die Verticalaxe eine Neigung von etw $21^{\circ}$ besilzt. Platten zur Messung des Axenwinkels liessen sich theils : wegen der geringen Dicke, theils wegen der Spaltbarkeit der Krystalle nicht herstellen.

Die Doppelbrechung ist gering und negativ.

Die Krystalle sind sehr vollkommén spaltbar nach der Symmetrieebene. Eine weniger vollkommene Spaltbarkeit nach dem Ortbopinakoid scheint, da die Spaltfläche eine dem Faserbruch des Gypses ähnliche Beschaffenheit zeigt, durch Alternation prismatischer Spaltflächen hervorgebrachi zu sein.

Die Härte ist ungefähr $2 \frac{1}{2}$.

Das specifische Gewicht wurde mittelst des Pyknometers in Oel bestimmt, da die Krystalle zu klein sind, um sie mil Erfolg mit Parafin zu uberziehen. Es wurde bei $13^{0} \mathrm{C}$. gefunden zu 2,1155.

In Beziehung auf die Löslichkeitsverhältnisse des Minerals ist zu erwähnen, dass das Sulfat in Wasser ausserordentlich leicht zerfliesslich ist. Die dicke, ölartige Flüssigkeit ist schwach gelb gefärbt, bat einen herben, adstringirenden Geschmack und trubt sich beim Erwärmen unter Abscheidung basischer Salze. 
Für die chemische Analyse wurden sehr reine Krystalle verwendet, welchen ebenso wie dem Coquimbit nur eine kleine Menge Quarzsand anhing (in den verschiedenen Analysen zwischen 1,5\% und 1,8\% schwankend).

Die nachstehende Analyse, der ich noch die nachträglich ausgefuhrte Controlbestimmung von $\mathrm{Fe}_{2} \mathrm{O}_{3}$ und $\mathrm{SO}_{3}$ beifuge, ist das Mittel aus vier Bestimmungen.

$\begin{array}{lcc} & & \text { Controlbestimmung } \\ \mathrm{SO}_{3} & 39,83 & 41,40 \\ \mathrm{Fe}_{2} \mathrm{O}_{3} & 27,66 & 27,59 \\ \mathrm{Al}_{2} \mathrm{O}_{3} & \mathrm{Spur} & \\ \mathrm{CaO} O & 0,40 & \\ \mathrm{MgO} & \mathrm{Spur} & \\ \mathrm{H}_{2} \mathrm{O} & 31,35 & \\ \text { Summe } & 99,24 & \end{array}$

Die Bestimmung des Wassers bei verschiedenen Temperaturgraden ergab folgendes Resultat:

Es entwichen

$$
\begin{array}{cc}
\text { bei } 100^{\circ} & 20,84 \% \\
-\quad 140 & 3,60 \\
-\quad 180 & 4,68 \\
-\quad 240 & 1,01 \\
\text { darúber } & 1,61
\end{array}
$$

Berechnet man aus der oben angefubrten Analyse die Aequivalentzahlen, so ergiebt sich für das Mineral die Formel $F_{2} S_{3} O_{12}+10$ aq, wie aus der nachstehenden Tabelle ersichtlich ist.

\begin{tabular}{ccccccccr} 
In 100 & \multicolumn{2}{c}{$\mathrm{SO}_{3}$} & \multicolumn{2}{c}{$\mathrm{Fe}_{2} \mathrm{O}_{3}$} & \multicolumn{2}{c}{$\mathrm{CaO}$} & \multicolumn{2}{c}{$\mathrm{H}_{2} \mathrm{O}$} \\
Theilen & $\%$ & Aequiv. & $\% \%$ & Aequiv. & $\%$ & Aequiv. & $\%$ & Aequiv. \\
Verlangt: & $\mathbf{4 1 , 3 8}$ & $\mathbf{5 1 7 3}$ & $\mathbf{2 7 , 5 8}$ & $\mathbf{1 7 2 4}$ & - & - & $\mathbf{3 1 , 0 4}$ & $\mathbf{1 7 2 4 4}$ \\
Gefunden: & $\mathbf{4 0 , 1 4}$ & $\mathbf{5 0 1 8}$ & $\mathbf{2 7 , 8 7}$ & $\mathbf{1 7 4 2}$ & $\mathbf{0 , 4 0}$ & $\mathbf{7 1}$ & $\mathbf{3 1 , 5 9}$ & $\mathbf{1 7 5 5 0}$
\end{tabular}

Das Salz enthält daher nur ein Molekul Wasser mehr als der Coquimbit. In seiner krystallographischen Ausbildung lässt das Mineral hingegen trotz der dem Coquimbit so nahe stehenden Zusammensetzung keinerlei Beziehung zu diesem erkennen.

Dem Sulfat, das offenbar ein neues Mineral ist, gebe ich zu Ehren meines früheren Lehrers, des um Geologie und Mineralogie so bochverdienten Professors Dr. F. A. v. Que nstedt, den Namen Quenstedtit. . 


\section{Copiapit (Haidinger).}

Den Namen Copiapit hat im Jahre 1845 Haidinger*) gegeben fur das von Meyen $n^{* *}$ ) von Copiapó mitgebrachte, von G. Rose $\left.e^{* *}\right)$ analysirte Salz. Nach $\mathrm{R}$ ose ist das in schwefelgelben Blättchen und Körnern als Ueberzug auf Goquimbit auftretende Mineral nach der Formel $\mathrm{2Fe}_{2} \mathrm{O}_{3}$. $5 \mathrm{SO}_{3}, 48 \mathrm{H}_{2} \mathrm{O}$ zusammengesetzt, und die kleinen Krystalle zeigen eine vollkommene Spaltbarkeit nach der Tafelläche.

Eine weitere Analyse desselben Vorkommens finden wir bei Domeyko t). Dieselbe stimmt sehr gut mit der Rose's überein. Ich werde auf diese wie auf jene später noch einmal zurúckkommen.

Ueber das Krystållsystem des Copiapit ist bis jetzt etwas Sicheres nicht bekannt geworden; bald nahı man für ihn das hexagonale, bald das rhombische System in Anspruch. B ertrand $+\dagger)$, welcher die kleinen Kryställchen mit dem Mikroskop untersuchte, fand, dass dieselben rhombisch krystallisiren, tafelförmig nach der Basis sind, die Formen $\{001\} 0 P$, $\{110\} \infty P,\{010\} \infty \breve{P} \infty,\{100\} \infty \bar{P} \infty$ zeigen, und dass der Prismenwinkel $102^{\circ}$ beträgt. Nach Bertrand ist ferner das Brachypinakoid Axenebene und die Verticalaxe erste Mittellinie; die Dispersion ist $\varrho>v$, und der Gharakter der Doppelbrechung negativ. Des Cloizeaux $+f+$ ) hat die Angaben von Bertrand gepruft und gefunden, dass die Verticalaxe nicht erste, sondern zweite Mittellinie ist und die Dispersion nicht $\varrho>v$, sondern $\varrho<v$. Ausserdem hat er den stumpfen Axenwinkel in Oel gemessen und gefunden:

$$
2 H_{0}=\left\{\begin{array}{l}
113^{0} 10 \frac{1^{\prime}}{2} \text { für rotbes Glas, } \\
114 \quad 15 \text { für } \mathrm{Na} \text {-Licht. }
\end{array}\right.
$$

Die von mir untersuchten Kryställchen sind schwefelgelb gefärbt und durchsichtig; sie bilden öfters lose Aggregate und erreichen eine Grösse bis zu $2 \frac{1}{2} \mathrm{~mm}$ in der Länge und Breite und höchstens $\frac{1}{2} \mathrm{~mm}$ in dẹr Dicke. Zum Theil sind sie mit recht glänzenden Flächen versehen und lassen Messungen mit dem Reflexionsgoniometer zu, welche, wenn auch wegen starker Längsstreifung nicht uberall befriedigend genau, doch ein Bestimmen der krystallographischen Constanten recht wobl gestatten. Nach meinen Untersuchungen krystallisirt der Gop i a i t

*) 1. c. (10) 489 .

**) 1. c. (1).

***) 1. c. (2) 314 .

t) 1. c. (15) 155 .

tf) 1. c. (11) 11. In dem Referat über diese Arbeit in dieser Zeitschr. ist wobl irrthümlicher Weise angegeben zweite Miltellinie $\perp$ auf $0 P$ und Dispersion $\varrho<v$.

t十t) l. c. (12) 41 . 
I. Beitrag zur Kenntniss der Sulfate von Tierra amarilla bei Copiapó in Chile. 15

Monos ymmetris ch.

$$
\begin{gathered}
a: b: c=0,4791: 1: 0,9759 \\
\beta=71{ }^{\circ} 56^{\prime} .
\end{gathered}
$$

Die Krystalle stellen im Wesentlichen drei verschiedene Typen dar. An allen dreien ist die vorherrschende Fläche die Symmetrieebene $b=$ $\{010\} \infty R \infty$, nach welcher die Krystalle auch tafelförmig ausgebildet sind.

Der einfachste Typus (Fig. 7) zeigt ausser $b=\{010\} \infty R \infty$ die Flächen $m=\{110\} \infty P, s=\{015\} \frac{1}{5} R \infty, o=\{\overline{4} 49\}+\frac{4}{9} P$.

Flächenreicher ist gewöhnlich der zweite Typus (Fig. 8). Er unterscheidet sich von dem vorhergehenden durch das Auftreten von negativen Hemipyramiden. Gewöhnlich beobachtet man die Formen $b=\{010\} \infty R \infty$, $m=\{110\} \infty P, p=\{120\} \infty R 2, q=\{011\} R \infty, r=\{023\}_{3}^{2} R \infty, s=$ $\{015\} \frac{1}{5} R \infty, d=\{\overline{4} 09\}+\frac{4}{9} P \infty, o=\{\overline{4} 49\}+\frac{4}{9} P, x=\{427\}-\frac{4}{7} P 2$.

Der dritte Typus endlich zeichnet sich durch das Auftreten mehrerer verscbiedener abgeleiteter Pyramiden aus. Die Fig. 9 zeigt einen verhältnissmässigen einfachen Krystall dieses Typus mit den Formen: $b= \begin{cases}010 \\ 0\end{cases}$ $\infty R \infty, m=\{110\} \infty P, s=\{015\} \frac{1}{5} R \infty, x=\{427\}-\frac{4}{7}+2, n=\{\overline{7} .4 .28\}$ $+\frac{1}{4} 7 \frac{7}{7}, y=\{T \overline{5} .2 .18\}+\frac{5}{6}+\frac{15}{2}$.

Als seltenere und auch meist schlecht entwickelte Formen wurden ferner an verschiedenen Krystallen noch folgende Flächen beobachtet: $\{580\} \infty R \frac{8}{5},\{7.20 .0\} \infty R_{\frac{20}{7}},\{4.15 .0\} \infty R_{\frac{15}{4}},\{4.17 .0\} \infty R \frac{17}{4},\{750\} \infty P \frac{7}{5}$, $\{730\} \infty P \frac{7}{3},\{920\}-\infty P \frac{9}{2},\{127\}-\frac{2}{7} R 2,\{\overline{4} .12 .27\}+\frac{4}{9} R 3,\{\overline{2} \overline{0} .12 .27\}+\frac{20}{2} 7 \frac{5}{3}$.

\begin{tabular}{|c|c|c|c|c|}
\hline \multirow{3}{*}{$\begin{array}{l}b: m= \\
b: q=\end{array}$} & & \multicolumn{2}{|c|}{ Gemessen : } & Berechnet: \\
\hline & $(0 \mathrm{~T} 0):(1 \bar{T} 0)$ & $={ }^{*} 65^{0} 3$ & $30^{\prime}$ & - \\
\hline & $(0 T 0):(0 T 1)$ & $=* 47$ & 9 & - \\
\hline$m: q=$ & $(110):(011)$ & $={ }^{*} 60 \quad 4$ & 49 & $一$ \\
\hline$b: r=$ & $(010):(023)$ & $=585$ & 50 & $58^{0} 16^{\prime}$ \\
\hline$b: s=$ & $(010):(015)$ & $=792$ & 24 & 7929 \\
\hline$b: p=$ & $(010):(120)$ & $=484$ & 42 & 4740 \\
\hline$b: d=$ & $(010):(\overline{4} 09)$ & $=901$ & 10 & $90 \quad 0$ \\
\hline$b: 0=$ & $(010):(\overline{4} 49)$ & $=70$ & 6 & 6949 \\
\hline$b: n=$ & $(010):(\overline{7} .4 .28)$ & $=822$ & 29 & 8214. \\
\hline$b: y=$ & $(010):(\overline{15} .2 .18)$ & $=862$ & 27 & 8630 \\
\hline$b: x=$ & $(010):(427)$ & $=812$ & 23 & 8125 \\
\hline$s: n=$ & $(015):(\overline{7} .4 .28)$ & $=283$ & 37 & 2938 \\
\hline$s: 0=$ & $(015):(\overline{4} 49)$ & $=383$ & 32 & 3840 \\
\hline$n: y=$ & $.4 .28):(\pi \overline{5} .2 .18$ & $=454$ & 44 & $44 \quad 40$ \\
\hline
\end{tabular}

Die gemessenen und berechneten Winkel ${ }^{*}$ ) sind folgende:

*) Die Winkel sind hier wie im Folgenden meist an verschiedenen Krystallen mehrfach gemessen und aus den Messungen ist das Mittel genommen. 


$\begin{array}{llr} & \text { Gemessen: } & \text { Berechnet: } \\ (010):(4.17 .0) & =26^{0} 49^{\prime} & 27019^{\prime} \\ (010):(4.15 .0) & =3026 & 3021 \\ (010):(7.20 .0) & =3820 & 3732 \\ (010):(580) & =5355 & 5353 \\ (010):(750) & =7156 & 7159 \\ (010):(730) & =7926 & 7857 \\ (010):(920) & =8416 & 8413 \\ (010):(\overline{2} 0.12 .27)=7742 & 7734 \\ (010):(4.12 .27)=4140 & 4212 \\ (010):(127) & =5747 & 5851\end{array}$

Selten ist eine Form mit all' ihren Flächen vorhanden, doch ist eine Regelmässigkeit in dem Fehlen oder Auftreten nicht zu beobachten.

Was die oplischen Verhältnisse anlangt, so habe ich in Uebereinstimmung mit Des Cloizea ux gefunden, dass die zweite Mittellinie senkrecht steht auf der Tafelfläche $\{010\} \infty R \infty$ und dass die Axenebene ungefähr mit dem Hemidoma $\{\overline{4} 09\}+{ }_{9}^{4} P_{\infty}$ zusammenfällt. Nur die Grösse des stumpfen Axenwinkels weicht elwas von der von Des Cloizeaux beslimmten ab. Ich fand in Oel

$$
2 H_{0}=111^{0} 36^{\prime} \text { für } \mathrm{Na} \text {-Licht. }
$$

Es gelang nicht, den spitzen Axenwinkel zu messen.

Die Doppelbrechung ist schwach negativ.

Der Pleochroismus ist sehr deutlich und zwar sind die parallel a schwingenden Strahlen dunkel schwefelgelb, die parallel $\mathfrak{b}$ schwingenden farblos bis gelblich und die nach $c$ schwingenden grünlichgelb. Die Absorption ist $\mathfrak{a}>\mathfrak{c}>\mathfrak{b}$.

Der Copiapit ist vollkommen spaltbar nach der Symmetrieebene $\{010\} \infty R \infty$ und viel unvollkommener nach $\{409\}+\frac{4}{9} P \infty$.

Seine Härte ist $2 \frac{1}{2}(-3)$. Das specifische Gewicht wurde mittelst des Pyknometers in Oel bei $13^{\circ} \mathrm{C}$. bestimmt zu 2,103.

Das Mineral ist in wenig Wasser leicht zu einer dicken, gelblich gefärbten Flüssigkeit löslich. Die Lösung reagirt sauer, hat einen herben, wenig zusammenziehenden Geschmack und trubt sich beim Kochen.

Die erste chemische Untersuchung verdanken wir, wie schon oben bemerkt, G. Rose*). Derselbe fand, dass die Zusammensetzung des Copiapit durch die Formel $2 \mathrm{Fe}_{2} \mathrm{O}_{3} .5 \mathrm{SO}_{3}+18$ aq ausgedruckt werde. $\mathrm{Ram}-$ melsberg**) dagegen bat aus derselben Analyse die Formel $\mathrm{Fe}_{4} \mathrm{~S}_{5} \mathrm{O}_{21}$

*) l. c. (2) 314 .

**) 1. c. (19) 1, 276. 
+13 aq resp. $\left\{\begin{array}{c}5 \mathrm{Fe}_{2} \mathrm{~S}_{3} \mathrm{O}_{12} \\ \mathrm{H}_{6} \mathrm{Fe}_{2} \mathrm{O}_{6}\end{array}\right\}+36$ aq abgeleitet, wofur $\mathrm{Gr}$ oth $\left.{ }^{*}\right)$ in seinen Tabellen $\mathrm{Fe}_{4}(\mathrm{HO})_{2}\left(\mathrm{SO}_{4}\right)_{5} \cdot 11$ aq geschrieben hat. Eine weitere Analyse des Sulfates haben wir von Dom e y k o**). Ich lasse die beiden Analysen unten mit der meinigen folgen. Die letztere ist wieder mit sehr reinem, krystallisirtem Material ausgeführt und das Mittel aus mehreren Analysen, bei welchen der unlösliche bis 1,6\% betragende Quarzsand von der Substanz in Abzug gebracbt ist. Ferner ist noch eine nachträglich ausgeführte Controlbestimmung der Schwefelsäure und des Eisens beigefúgt.

\begin{tabular}{|c|c|c|c|c|}
\hline & Rose: & Dome yko: & Linck: & Controlbestimmung: \\
\hline $\mathrm{SO}_{3}$ & 39,60 & 38,00 & 38,91 & 40,49 \\
\hline $\mathrm{Fe}_{2} \mathrm{O}_{3}$ & 26,11 & 24,66 & 30,10 & 30,84 \\
\hline $\mathrm{Al}_{2} \mathrm{O}_{3}$ & 1,95 & 1,16 & Spur & \\
\hline $\mathrm{MgO}$ & 2,64 & 0,84 & - & \\
\hline $\mathrm{CaO}$ & 0,06 & 1,39 & Spur & \\
\hline $\mathrm{SiO}_{2}$ & 1,37 & 5,20 & 一 & \\
\hline $\mathrm{H}_{2} \mathrm{O}$ & 29,67 & 28,74 & 30,74 & \\
\hline Summe & 101,40 & 99,99 & 99,75 & \\
\hline
\end{tabular}

Bezuglich der Fluchtigkeit des Wassers hat sich ergeben :

$$
\begin{array}{cc}
\text { Es entwichen bei } 110^{\circ} \text { C. } & 12,73 \% \\
\text { weiter }-160 & 6,59 \\
-\quad-210 & 6,39 \\
-\quad-270 & 2,43 \\
-\quad-360 & 0,64 \\
\text { und daruber } & 1,29
\end{array}
$$

Berechnen wir aus den obigen Analysen die Aequivalentverbälnisse und stellen sie der Uebersicht halber in einer Tabelle zusammen, so erhalten wir:

$\begin{array}{lrrr} & \text { Rose: } & \text { Domeyko: } & \text { Linck: } \\ \mathrm{SO}_{3} & 4950 & 4750 & 4864 \\ \mathrm{Fe}_{2} \mathrm{O}_{3} & 1632 & 1541 & 1881 \\ \mathrm{Al}_{2} \mathrm{O}_{3} & 191 & 113 & - \\ \mathrm{MgO} & 660 & 210 & - \\ \mathrm{CaO} & 11 & 250 & - \\ \mathrm{H}_{2} \mathrm{O} & 16483 & 15967 & 17078\end{array}$

Aus dieser Zusammenstellung ist leicht ersichtlich, dass der Copiapit

*) 1. c. (14) 55 .

**) 1. c. (15) 155 .

Groth, Zeitschrift f. Krystallogr, $X V$. 
zweifellos ein basisches Sulfat ist; es erscheint am nalurlichsten die Annahme von Grotb, dass ein. Theil der Valenzen des Eisenoxyds durch Hydroxylgruppen gesättigt sei.

Der Wassergehalt, wie ihn Rose und Domeyko gefunden haben, stimmt nicht mit meiner Analyse úberein. Aber aus der Beschreibung des Ersteren und aus dem hohen Gehalt an $M g O$ und $C a O$ lässt sich bei $\mathrm{R}$ os e sowohl wie bei Domeyko wohl schliessen, dass keiner der Beiden ein reines und frisches Material analysirte. Bei Rose tritt, wie es bei verwitterlem Material zu erwarten, eine Anreicherung von Eisenoxyd und Wasserverlust ein, während bei Domeyko nur elwa $3 \%$ Wasser fehlen.

Diese Verhältnisse waren auch Veranlassung, dass Rammelsberg und Groth einen geringeren Wassergebalt annahmen. Der Erstere berechnet $13 \mathrm{H}_{2} \mathrm{O}$ auf $\mathrm{Fe}_{4} \mathrm{~S}_{5} \mathrm{O}_{21}$, der Letztere $11 \mathrm{H}_{2} \mathrm{O}$ fur die Formel $\mathrm{Fe}_{4}(\mathrm{HO})_{2}$ $\left(\mathrm{SO}_{4}\right)_{5}$. Meine Analyse giebt $18 \mathrm{H}_{2} \mathrm{O}$ und ich darf annehmen, dass diese Analyse richtig ist, denn es hat auch G. R os e jedenfalls mit Rücksicht auf sein zum Theil zerselztes Material die Formel $\mathrm{Fe}_{4} \mathrm{~S}_{5} \mathrm{O}_{21}+18$ aq angenommen. Ich neige zu der Annahme, dass sogar 19 Theile $\mathrm{H}_{2} \mathrm{O}$ vorhanden sind und möchte für den Copiapit die Formel $\mathrm{Fe}_{4}\left(\mathrm{HO}_{2}\left(\mathrm{SO}_{4}\right)_{5}+18\right.$ aq vorschlagen. Die Flüchtigkeitsverhältnisse des Wassers in dem Mineral widersprechen zum Mindesten der Annahme der letztgenannten Formel nicht.

In 100 Gewichtstheilen verlangt

\begin{tabular}{llll}
\multicolumn{2}{c}{ die Formel : } & \multicolumn{2}{c}{ wurden gefunden : } \\
$\mathrm{SO}_{3}$ & 37,665 & $\mathrm{SO}_{3}$ & 39,00 \\
$\mathrm{Fe}_{2} \mathrm{O}_{3}$ & 30,132 & $\mathrm{Fe}_{2} \mathrm{O}_{3}$ & 30,18 \\
$\mathrm{H}_{2} \mathrm{O}$ & 32,203 & $\mathrm{H}_{2} \mathrm{O}$ & 30,89
\end{tabular}

Die Differenz ist also eine sehr geringe und kann ebensowohl im Material, als auch in den Analysenfehlern begründet sein.

Ausser in Tierra amarilla, wo der Copiapit als krystallinischer Ueberzug auf dem Coquimbit vorkommt, findet sich derselbe nach $\mathrm{Hoh}$ man ${ }^{*}$ ) N. von Sierra Gérrda bei Garacoles in Bolivieu. Hier bildet er grünlichgelbe, derbe, krystallinische Massen, in welchen Hohman$\mathrm{n}$ it und $\mathrm{Amar}$ an $\mathrm{tit}$ eingesprengt vorkommen. Die Massen bestehen aus lauter kleinen Kryställchen, welche einem der von mir oben beschriebenen Typen angehören.

Zum Copiapit gehört auch der Misy vom Rammelsberge bei Goslar. Diese Varietät des Minerals bildet ein lockeres, schwefelgelbes Aggregat von kleinen Kryställchen, welche alle die oben erwähnten Eigenschaften des Copi a pit besitzen und gewöhnlich dem ersten Typus (Fig. 7)

*) l. c. (9) bei Frenzel. 
angehören. Der Copiapit vom Rammelsberge wurde analysirt von Borcher.s*), List**) und Ahrend und Ulrich***).

Eine Analyse der beiden Letzteren, welche mit deutlich krystallinischem $M$ is y angestellt wurde, ergab:

\begin{tabular}{|c|c|c|c|c|}
\hline $\mathrm{SO}_{3}$ & $39,4.40$ & $=$ & 4930 & Aequiv. \\
\hline $\mathrm{Fe}_{2} \mathrm{O}_{3}$ & 28,00 & $=$ & 1750 & - \\
\hline $\operatorname{ZnO}$ & 2,00 & $=$ & 247 & - \\
\hline $\mathrm{H}_{2} \mathrm{O}$ & 30,64 & $=$ & 17022 & - \\
\hline
\end{tabular}

Daraus berechnet Ulri ch dieselbe Formel, wie sie R ose für den Copiapit gefunden hat. Somit sprechen chemische Zusammensetzung und Krystallform unzweifelhaft für die Identität des Mis y mit dem Copiapit.

\section{Stypticit (Hausmann).}

Im Jahre 1841 hat Pride a u $\mathrm{t}_{\text {) }}$ ) ein von Copiapó stammendes, feinfaseriges, basisches Eisenoxydsulfat analysirt und demselben den Namen Fibroferrit gegeben. Er fand das Mineral bestehend aus $\mathrm{SO}_{3} 26 \%$, $\mathrm{Fe}_{2} \mathrm{O}_{3} 31 \%, \mathrm{H}_{2} \mathrm{O} 33 \%, \mathrm{~S}$, Erde und Verlust $10 \%$ und leitete daraus, eigentlich ohne Berechtigung, die Formel $2 \mathrm{Fe}_{2} \mathrm{O}_{3}$. $3 \mathrm{SO}_{3} \cdot 18 \mathrm{H}_{2} \mathrm{O}$ ab.

Sechs Jahre später hat $\mathrm{H}$ a us man $\mathrm{n}+\dagger$ ) für das von Meyen in Tierra a m a rill a gefundene, von G. R ose ttt) analysirte, gelblichgrune, fein radialfaserige, basische schwefelsaure Eisenoxyd den Namen Stypticit eingeführt; wie Hausmann selbst sagt, nur deshalb, weil die Analyse G. Rose's nicht mit der von Pridea u übereinstimmt. Rose fand nämlich für die Zusammensetzung folgende Zahlen: $31,73 \%, S O_{3}, 28,11 \%$ $\mathrm{Fe}_{2} \mathrm{O}_{3}, 1,91 \% \mathrm{CaO}, 0,59 \% \mathrm{MgO}, 1,43 \% \mathrm{SiO}_{2}, 36,56 \% \mathrm{H}_{2} \mathrm{O}$ und hieraus berechnete er die Formel $\mathrm{Fe}_{4} \mathrm{~S}_{4} \mathrm{O}_{18}+21$ aq, während $\mathrm{H}$ a u s mann* und Rammelsberg* ${ }^{*}+f$ ) auf Grund der Analyse von Prideaux fur den Fibroferrit von Copiapó die Formel $\mathrm{Fe}_{6} \mathrm{~S}_{5} \mathrm{O}_{24}+27$ aq aufstellten.

Die von mir an möglichst reinem und vollständig frischem Material ausgeführte Analyse ergab folgende Werthe :

*) Vergl. I. c. (16) bei Ulrich.

**) Desgl. I. c. (16) bei Ulrich und $L$ is $t(25)$.

***) 1. c. (16).

t) 1. c. (17) 397 .

t†) 1. c. (18) 2, II, 1203 .

t十†) 1. c. (2) 316 .

*) I. c. (18) 2, II, 1203.

*十†) 1. c. (30) 277. 


$$
\begin{aligned}
& \text { In } \mathrm{HCl} \text { unlöslich } \quad 0,63 \% \\
& \mathrm{SO}_{3} \quad 32,94=4118 \text { Aequiv. } \\
& \mathrm{Fe}_{2} \mathrm{O}_{3} 32,43=2027 \text { - } \\
& \mathrm{Al}_{2} \mathrm{O}_{3} \text { Spur = - - } \\
& \mathrm{CaO} \quad 0,40=72 \text { - } \\
& M g O \text { Spur }=- \\
& \mathrm{H}_{2} \mathrm{O} 34,32=19067 \quad- \\
& \text { Summe 100,72 }
\end{aligned}
$$

Die Fluchtigkeitsverhältnisse*) des Wassers sind folgende :

$$
\begin{array}{cc}
\text { Es entweichen bei } 110^{\circ} \text { C. } & 21,37 \% \\
\text { weiter }-155 & 5,52 \\
-\quad-210 & 4,15 \\
-\quad-260 & 2,59 \\
\text { und daruber } & \left.1,57^{*}\right)
\end{array}
$$

Ich schliesse aus diesen Resultaten mit denselben Grunden, wie ich sie beim Copiapit auseinandergesetzt habe, dass die Zusummensetzung des Stypticit am wahrscheinlichsten der Formel $\mathrm{Fe}_{2}(\mathrm{OH})_{2} \mathrm{~S}_{2} \mathrm{O}_{8}+9$ aq resp. $\mathrm{SO}_{2} \mathrm{O}_{\mathrm{O}}^{\mathrm{O}}>\mathrm{Fe}-\mathrm{OH}+4 \frac{1}{2} \mathrm{H}_{2} \mathrm{O}$ entspricht; eine Annahme, welche durch die Analysen entschieden gerechtfertigt sein düfte.

Die obige Formel weicht von derjenigen, wie sie Groth in seinen Tabellen gegeben hat, nur wenig im Wassergehalt ab. Sie erfordert etwas weniger Wasser als die von $G$ roth und wenig mehr als die von G. Rose aufgestellte,

$$
\begin{array}{lcc}
\multicolumn{2}{r}{\text { indem sie in } 100 \text { Theilen }} & \text { während gefunden **) } \\
\text { verlangl: } & \text { wurden : } \\
\mathrm{SO}_{3} & 32,00 & 32,73 \\
\mathrm{Fe}_{2} \mathrm{O}_{3} & 32,00 & 32,66 \\
\mathrm{H}_{2} \mathrm{O} & 36,00 & 34,61
\end{array}
$$

Das Feblen von 1,4\% Wasser kann bei einem so leicht verwitterbaren Mineral nicht als Beweis gegen meine Annahme aufgefasst werden.

Ausser von Rose wurde der Stypticit von Copiapó analysirt von Smith***), Tobler f) und Field $t+$ ). Sie verwendeten alle recht frisches

*) Die Angaben Brun's (l. c. 19), dass $8 \%$ Wasser erst bei dunkler Rothgluth flüchtig seien, ist jedenfalls ganz falsch und daher ebenso dessen Formel $\mathrm{Fe}_{2} \mathrm{~S}_{2} \mathrm{H}_{4} \mathrm{O}_{11}$ +8 aq. Das Wasser entweicht längst, ebe die Substanz in's Glüben gerăth, und später entweicht nur noch Schwefelsäure. Richtiger ist die Angabe Field's (1. c. 20), nach welchem 3 Moleküle Wasser erst bei 5000-6000 F. flüchtig sind.

**) Auf 100 berechnet nach Abzug des $\mathrm{CaO}$ als Gyps.

***) l. c. (21).

t) 1.c. (22).

tt) l. c. $(20)$. 
Material und ihre Analysen stimmen daher sehr gut mit den theoretisch geforderten Verhältnissen überein. So berechnet Field die Formel $F_{4} S_{4} O_{18}$ +20 aq, während $\mathrm{Sm}$ ith die Formel $\mathrm{Fe}_{4} \mathrm{~S}_{4} \mathrm{O}_{18}+21$ aq giebt. Zum Vergleiche lasse ich die entsprechenden Analysen hier folgen.

\begin{tabular}{|c|c|c|c|c|}
\hline & \multicolumn{2}{|c|}{ Smith: } & Tobler: & Field: \\
\hline Unlöslich & 0,54 & 一 & - & - \\
\hline $\mathrm{SO}_{3}$ & 30,25 & 30,42 & 31,49 & 31,94 \\
\hline $\mathrm{Fe}_{2} \mathrm{O}_{3}$ & 31,75 & 30,98 & 31,69 & 31,89 \\
\hline $\mathrm{H}_{2} \mathrm{O}$ & 38,20 & unbest. & $36,82 * j$ & 35,90 \\
\hline Summe & 100,74 & & 100,00 & 99,73 \\
\hline
\end{tabular}

Der Stypticit von Tierra amarilla bei Copiapó, wie er mir vorliegt, bildet bald mehr oder weniger breit gedruckte cylindrische, bald verästelte und wurmartig gekrummte, radialfaserige Aggregate von gelblichgrüner Farbe und lebhaftem seidenartigen Glanz. Die Cylinder zeigen meist im Innern einen Hohlraum und bestehen öfters aus mehreren bis $\boldsymbol{5} \mathbf{~ m m}$ dicken Schalen. An der Innenseite der Cylinder hat das Mineral eine dunkelgrune bis schwarze Farbe. Die Fasern sind sehr fein und lassen sich ähnlich wie Asbest zu einer voluminösen Wolle zerzausen.

In Wasser, besonders in warmem Wasser, quilt der Stypticit sehr stark auf und wild unter Abscheidung brauner Producte zersetzt. Die uberstehende Flitssigkeit reagirt sauer und hat einen herben, zusammenziehenden Geschmack.

Die Härte des Minerals ist $(2-) 2 \frac{1}{2}$. Sein specifisches Gewicht, welches mittelst des Pyknometers in Oel bestimmt wurde, ist gleich 1,857**) bei $13^{\circ} \mathrm{C}$.

Unter dem Mikroskope erscheinen die sebr dünnen Fasern fast farblos; sie sind am Ende entweder schräg abgestumpft, oder sie zeigen eine dachförmige Endigung. Ein Theil der Fasern löschı zwischen gekreuzten Nicols im parallelen polarisirlen Lichte unter einer Neigung von etwa $10^{\circ}$ im Maximum gegen ihre Längsrichtung aus, während bei vielen derselben die Auslöschung mit der Längsrichtung zusammenfällt. Es ist daher wahrscbeinlich, dass der Stypticit in dem monosymmetrischen Systeme krystallisirt.

Bei der Zersetzung verschwindet zundchst der Seidenglanz und die gelblichgrüne Farbe geht in grünlichgrau und zuletzt in graugelb über. Es tritt also jedenfalls eine Anreicherung von Eisenoxyd ein. Ein solcher zersetzter Stypticit scheint, soweit man nach der Beschreibung schliessen kann, der von Prideaux untersuchte sogenannte Fibroferrit von Copi a pó gewesen zu sein.

*) Aus dem Verlust.

**) Von Smith (l. c. 21) zu 1,84 angegeben. 
Der einzige Fundort für Stypticit ausser Tierra amarilla bei Copiapó ist Pallières im Departement du Gard in Frankreich. Dieses Vorkommen, welches auch nicht ganz frisch ist, sondern Glanz und Farbe verloren resp. gewechselt hat, ist von Pis a ni*) untersucht worden. Derselbe fand:

$$
\begin{array}{ll}
\mathrm{SO}_{3} & 29,72 \\
\mathrm{Fe}_{2} \mathrm{O}_{3} & 33,40 \\
\mathrm{CaO} & \mathrm{Spur} \\
\mathrm{H}_{2} \mathrm{O} & 36,88 \text { aus der Differenz } \\
\cline { 2 - 2 } \text { Summe } & 100,00
\end{array}
$$

Es bat also auch hier schon eine Anreicherung von Eisenoxyd stattgefunden, aber noch nicht in so hohem Maasse, wie dies bei dem Fibroferrit Prideaux's der Fall ist.

\section{Römerit **) (Grailich).}

Das Mineral, im Jahre 1858 am R ammelsberge bei Goslar entdeckt und zu Ehren des damaligen Bergassessors A. Röm er benannt, wurde von Grailich***) der ersten krystallographischen Analyse unterworfen, während L. Tschermak $\dagger$ ) dessen erste chemische Untersuchung vornahm. Grailich mass die Winkel mit dem Anlegegoniometer und stellte den Römerit in das monosymmetrische System; Tschermak leitete aus seinen Analysen die Formel $\left(\mathrm{FeO} \mathrm{SO}_{3} . \mathrm{Fe}_{2} \mathrm{O}_{3} 3 \mathrm{SO}_{3}\right)+12 \mathrm{aq} \mathrm{ab}$.

Waren diese Krystalle zu wenig gut, um eine genaue Kenntniss der krystallographischen Eigenschaften der Substanz zu erlangen, so blieb J. B l a a s t†) die verdienstvolle Arbeit, an zwar kleinen, aber doch besseren Krystallen desselben Fundortes eine Untersuchung mit dem Reflexionsgoniometer durchzuführen. Seine Messungen ergaben, dass der Römerit nicht monosymmetrisch, sondern asymmetrisch krystallisirt, dass

$$
\begin{aligned}
& \alpha=89044^{\prime} \\
& \beta=10217 \\
& \gamma=8518
\end{aligned}
$$

und das Axenverhältniss $a: b: c=0,8791: 1: 0,8475$ sei. Aber auch diese Krystalle waren nicht so beschaffen, dass einigermassen auf Genauig-

*) 1. c. 23.

**) Dieses Mineral ist von mir in einer vorläufigen Mittheilung (1. c. 32) als "B ü ckin gi " " bezeichnet, nachträglich aber als identisch mit dem Römerit erkannt worden.

***) 1. c. (24).

t) l. c. (24) bei Grailich.

ti) I. c. (26). 
keit Anspruch gemacht werden konnte, wie sich aus den eigenen Auslührungen*) von Bla a s ergiebt.

Das reichliche Material von diesem Mineral, welches mir von dem für dasselbe neuen Fundorte 'Tierra amarilla bei C op i a pó vorlag, gestattete eine ziemlich vollständige Untersuchung.

Der Rö m eri t dieses Fundorles bildet kastanienbraune, krystallinische Massen, in deren Drusen bis $4 \mathrm{~mm}$ lange und bis 2 und $3 \mathrm{~mm}$ breite und dicke Krystalle von derselben Farbe sitzen. Diese Krystalle, welche allerdings nicht stets sehr glänzende Flächen haben und leicht trübe werden, dienten zur Bestimmung der krystallographischen Verhältnisse.

Auch ich fand in Uebereinstimmung mit Bla as, dass der Römeri t dem as ymmetrischen Krystallsystem angehört. Jedoch werde ich denselben etwas abweichend von B la as stellen, da hierdurch die Zeichen der Formen, an welchen die mir vorliegenden Krystalle reicher sind, einfacher werden. Die Spaltungsfläche, welche Bla as zum Brachypinakoid genomnen hat, nehme ich zur Basis, seine Pyramide zum Prisma, seine Brachydomenzone zur Zone der Makrodomen und seine Prismenzone zur Brachydomenzone.

Bei dieser Art der Aufstellung ergiebt sich :

$$
\begin{aligned}
a: b: c & =0,9682: 1: 2,6329 \\
\alpha & =116^{0} 2^{\prime} \\
\beta & =9441 \\
\gamma & =808
\end{aligned}
$$

Die Krystalle sind tafelförmig nach $c=\{001\} 0 P$. Ausserdem treten meist gross entwickelt auf: $x=\{10 \bar{T}\}+\bar{P} \infty, y=\{508\}-\frac{5}{8} \bar{P} \infty, q=$ $\{011\}, \breve{P}^{\prime} \infty$. Untergeordnet erscheinen die Flächen : $a=\{100\} \infty \bar{P} \infty, b=$ $\{010\} \infty \breve{P}_{\infty}, p=\{110\} \infty P^{\prime}, \quad m=\{320\} \infty \infty^{\prime} \breve{P}_{2}, q^{\prime}=\{01 \overline{1}\} \breve{P}^{\prime}, \infty, n=$

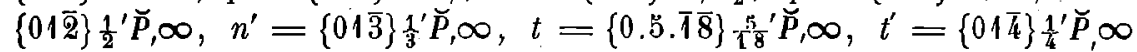
(Fig. 10). Diese Formen treten häufig fast alle gleichzeitig an demselben Krystalle auf. Seltener sind einfache Krystalle von der Combination $c=$ $\{001\} 0 P, x=\{10 T\}+\bar{P} \infty, y=\{508\}-\frac{5}{8} \bar{P} \infty, q=\{011\}, \breve{P}^{\prime} \infty, m=$ $\{110\} \infty P_{,}^{\prime}$. Die brachydiagonale Zone ist meist besonders flächenreich und die Flächen in der Richtung der Brachydiagonale gestreift. Nach der Axe a sind die Krystalle meist auch etwas verlängert.

Die gemessenen und berechneten Winkel sind folgende:

*) 1. c. (26) 1129 o. und 1124 u. 


\begin{tabular}{|c|c|c|c|}
\hline & Gemessen: & Berechnet & (Blaas): \\
\hline$c: a=$ & $(001):(100)={ }^{*} 89^{\circ} 36^{\prime}$ & - & $\left(88^{0} 55^{\prime}\right)$ \\
\hline$c: b=$ & $(001):(010)={ }^{*} 64 \quad 20$ & - & $(6250)$ \\
\hline$a: b=$ & $(100):(010)={ }^{*} 98 \quad 43$ & - & - \\
\hline$x: c=$ & $(10 T):(00 T)={ }^{*} 6827$ & 一 & $\left(\begin{array}{ll}68 & 12\end{array}\right)$ \\
\hline$b: p=$ & $(010):(110)={ }^{*} 4658$ & - & $(4721)$ \\
\hline$m: b=$ & $(320):(010)=3946$ & $39^{0} 56^{\prime}$ & - \\
\hline$y: c=$ & $(508):(001)=5626$ & $56 \quad 43$ & - \\
\hline$q: c=$ & $(011):(001)=4819$ & 4838 & $\left(\begin{array}{ll}46 & 38\end{array}\right)$ \\
\hline$q^{\prime}: c=$ & $(01 \bar{T}):(00 \bar{T})=9319$ & 9348 & $(94 \quad 45)$ \\
\hline$n: c=$ & $(01 \overline{\mathbf{2}}):(00 \bar{\top})=7116$ & $70 \quad 42$ & \\
\hline$n^{\prime}: c=$ & $(01 \overline{3}):(00 \overline{1})=5238$ & 5211 & \\
\hline$t: c=$ & $(0.5 . \overline{1} \overline{8}):(00 \bar{T})=4421$ & 4431 & \\
\hline$t^{\prime}: c=$ & $(01 \overline{4}):(00 \bar{T})=4046$ & $40 \quad 12$ & \\
\hline$n: a=$ & $(01 \overline{2}):(\overline{1} 00)=7950$ & $80 \quad 42$ & \\
\hline
\end{tabular}

Die optischen Verbältnisse konnten wegen der Kleinheit der Krystalle nicht so genau studirt werden, wie es wünschenswerth gewesen wäre, stimmen jedoch mit den Angaben Grailich's ziemlich vollständig uberein. Festgestellt wurde, dass die Axenebene so liegt, dass ibre Durchschnittsrichtung mil der Basis den von den Kanten $a: c$ und $b: c$ gebildeten stumpfen Winkel halbirt. Auf der Basis tritt eine Axe aus, und die erste Mittellinie ist etwa $30^{\circ}$ gegen die Normale auf $\{001\} 0 P$ geneigt. Der spitze Axenwinkel konnte an einem Blattchen gemessen werden und wurde in Oel gefunden :

$$
2 H_{a}=57045^{\prime} \text { fur } \mathrm{Na} \text {-Licht. }
$$

Die Doppelbrechung ist gering negativ, die Dispersion dagegen stark und zwar $\varrho>v$. Auch lässt sich auf der Basis Pleochroismus beobachten; die in der Axenebene schwingenden Strahlen sind röthlichbraun, die senkrecht dazu schwingenden gelblichweiss. In einem Bruchstuck, welches ungefähr senkrecht zu der ersten Mittellinie gesprungen war, traten beide Strahlen rotbbraun gefärbt aus. Die Absorplion ist $\mathfrak{b}>\mathfrak{a}=\mathfrak{c}$.

Die Spaltbarkeil des R ö me r it ist basisch, sehr vollkommen. Die Härte ist $3\left(-3 \frac{1}{2}\right)$.

Das specifische Gewicht wurde miltelst des Pyknometers in Oel bestimmt und ergab sich zu 2,102 bei $13^{\circ} \mathrm{G}$., während Grailich dasselbe zu 2,174 fand.

Das Sulfat ist in Wasser ausserordentlich leicht und vollkommen löslich; die Lösung reagirt stark sauer und hat eine braune Farbe. Beim Erwärmen scheiden sich basische Salze aus. Der Geschmack ist im Gegensalz zu den oben beschriebenen Eisenoxydsulfaten tintig und herb.

Zur Analyse wurde sehr reines Material des Minerals verwendet. Durch 
Auflösen in Wasser wurden durchschnitllich bis zu 2,66\% Quarzsand und Eisenkies davon getrennt. Die nachstehenden Zahlen sind das Mittel aus mehreren Bestimmungen, welchen ich noch eine Controlbestimmung von $\mathrm{SO}_{3}$ und $\mathrm{Al}_{2} \mathrm{O}_{3}+\mathrm{Fe}_{2} \mathrm{O}_{3}+\mathrm{FeO}$ beifuge.

Controlbestimmung :

$$
\begin{aligned}
& \mathrm{SO}_{3} \quad 38,47=4801 \text { Aequiv. } 39,22
\end{aligned}
$$

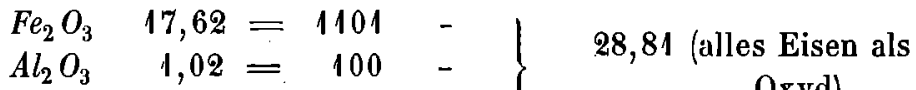

$$
\begin{aligned}
& \mathrm{FeO} \quad 9,06=1259-\} \quad \text { Oxyd) } \\
& \mathrm{CaO} \quad \text { Spur }=- \\
& \mathrm{H}_{2} \mathrm{O} \quad 34,10=18944 \quad
\end{aligned}
$$

Wenn man die Thonerde als das Eisenoxyd vertretend annimmt, so berechnet sich aus obigen Zablen die Zusammensetzung des Röm e rit als der Formel

$$
\mathrm{Fe}(\mathrm{Fe}, \mathrm{Al})_{2} \mathrm{~S}_{4} \mathrm{O}_{16}+15 \mathrm{aq}
$$

entsprechend. Sie verlangt in 100 Gewichtslbeilen :

$\begin{array}{lr}\mathrm{SO}_{3} & 38,929 \\ \mathrm{Fe}_{2} \mathrm{O}_{3} & 19,465 \\ \mathrm{FeO} & 8,759 \\ \mathrm{H}_{2} \mathrm{O} & 32,847\end{array}$

Tschermak dagegen fand in seiner Analyse :

$$
\begin{aligned}
& \text { Unlöslich } 0,50 \\
& \mathrm{SO}_{3} \quad 41,54=5192 \text { Aequiv. } \\
& \mathrm{Fe}_{2} \mathrm{O}_{3} \quad 20,63=1289 \quad \text { - } \\
& \mathrm{FeO} \quad 6,26=870- \\
& \text { ZnO } 1,97=243- \\
& \mathrm{CaO} \quad 0,58=103- \\
& \mathrm{H}_{2} \mathrm{O} \quad 28,00=15556 \quad- \\
& \text { Summe } 99,48
\end{aligned}
$$

Daraus berechnete er die Formel

$$
(\mathrm{Fe}, \mathrm{Zn}) \mathrm{Fe}_{2} \mathrm{~S}_{4} \mathrm{O}_{16}+12 \mathrm{aq} .
$$

Ein beträchtlicher Unterschied zwischen del Analyse von Tschermak und der meinigen ist also nur bezugglich des Wassergehaltes vorhanden. Es hebt jedoch Grailich ausdrücklich hervor, dass der Rö meri t vom Rammelsberge reichlich Blättchen von Gopiapit (Misy) einschliesse, was eine Abnahme des Gesammtwassergehaltes veranlassen muss.

Von einer erneuten vergleichenden Untersuchung der beiden einzigen 
Vorkommnisse vom Rammelsberge bei Goslar und von Gopiapó wird die Entscheidung abhängen, ob zwei verschiedene. Mineralien vorliegen oder der Wassergehalt des Römerit vom ersteren Fundorte höher ist.

Dieselbe Untersuchung wird dann auch daruber Auskunft geben, ob der B otr yogen, wie es Blaas*) vermuthet, mit dem Römerit in chemischer Beziehung identisch und eine dimorphe Modification derselben Substanz ist oder ob sich beide durch den Wassergehalt unterscheiden.

\section{Halotrichit (Glocker).}

Mit diesem Namen belegte Glocker**) im Jahre 1839 ein weisses haarförmiges Mineral, welches von Berthier****) (von unbekanntem Fundorte) und von Rammelsberg t) (von Mörsfeld in Rheinbayern) analysirt worden war. Aus ihren Analysen berechnete Glocker die Formel $\mathrm{Fe} \mathrm{Al}_{2} \mathrm{~S}_{4} \mathrm{O}_{16}+24$ aq.

Das mir vorliegende Material ist von weisser Farbe und feinfaserig und hat Seidenglanz. Die Fasern sind bis $5 \mathrm{~cm}$ lang und biegsam. Unter dem Mikroskop löschen sämmtliche Fasern schief gegen ihre Längsrichtung aus, woraus geschlossen werden dürfte, dass dieselben dem asymmetrischen Krystallsysteme angehören.

Es ist in kaltem Wasser vollkommen und leicht löslich zu einer farblosen Flüssigkeit, welche sauer reagirt und einen der Tinte ähnlichen zusammenziehenden Geschmack besitzt.

Die Härte des Minerals ist ungefähr $2 \frac{1}{2}$; sein specifisches Gewicht wurde mittelst des Pyknometers in Oel bestimmt zu 1,885 bei $13^{\circ} \mathrm{C}$. Resultat:

Die Analyse von reinem und ganz frischem Material lieferte folgendes

$\begin{array}{lrlrl}\mathrm{SO}_{3} & 33,98 & = & 4249 & \text { Aequiv. } \\ \mathrm{Al}_{2} \mathrm{O}_{3} & 10,43 & = & 1023 & - \\ \mathrm{Ti}_{2} \mathrm{O}_{3} & 0,95 & = & 64 & - \\ \mathrm{FeO} & 5,55 & = & 771 & - \\ \mathrm{CaO} & 0,69 & = & 122 & - \\ \mathrm{MgO} & 0,78 & = & 195 & - \\ \mathrm{H}_{2} \mathrm{O} & 46,94 & =26022 & - \\ \text { Summe } & 99,32\end{array}$

Hieraus berechnet sich die Formel

$$
\mathrm{Fe} \mathrm{Al}_{2} \mathrm{~S}_{4} \mathrm{O}_{16}+24 \text { aq }
$$

\#) 1. c. (31) 163 .

**) 1. c. (27) 694 .

***) 1. c. (28).

t) Vgl. l. c. (30) 288 . 
wie sie von Glocker angegeben wurde. Sie verlangt in 100 Theilen:

$\begin{array}{lr}\mathrm{SO}_{3} & 34,557 \\ \mathrm{Al}_{2} \mathrm{O}_{3} & 11,01: 3 \\ \mathrm{FeO} & 7,775 \\ \mathrm{H}_{2} \mathrm{O} & 46,653\end{array}$

Dabei habe ich allerdings den auffallenden Gehalt an Tilansäure, welche beim Kochen der sebr verdunnten Lösung unter Zusatz von $\mathrm{SO}_{2}$ ausfällt, als $\mathrm{Ti}_{2} \mathrm{O}_{3}$ berechnet und zu Thonerde gezogen, während ich Kalk und Magnesia als das Eisenoxydul vertretend aufgeführt habe. Es würde also die Formel genauer lauten:

$$
\left\{\begin{array}{l}
(\mathrm{Fe}, \mathrm{Mg}, \mathrm{Ca}) \mathrm{SO}_{3} \\
(\mathrm{Al}, \mathrm{Ti})_{2} \mathrm{~S}_{3} \mathrm{O}_{12}
\end{array}\right\}+12 \mathrm{aq} .
$$

Für den Halotrichit sind bis jetzt folgende Fundorte bekannt*): Mörsfeld in Rheinbayern, Solfatara von Pozzuoli, Björkbakka$\mathrm{g}$ ard in Finnland, Oroomiah in Persien. Hierzu kommt als fünfter Fundort Tierra a marilla bei Copiapó.

Hierzu kommen noch einige Salze, welche nichts besonders Bemerkenswerthes darbieten, nämlich:

7. Eisenvitriol (Melanterit),

8. Eisen-haltiger Kupfervitriol,

9. Keramohalit $\left(\mathrm{Al}_{2} \mathrm{~S}_{3} \mathrm{O}_{12}+18 \mathrm{aq}\right)$,

10. Gyps und

11. kleine gelbe, wahrscheinlich rhombische Kryställchen, über die wegen Mangel an genugendem Material Untersuchung und Mitheilung vorläufig nnterbleiben muss.

Die in der vorliegenden Abhandlung beschriebenen Salze verdanken, wie schon Meyen hervorgehoben hat und nach ihm mehrere Gelehrte bestätigten, ihre Entstehung der Zersetzung von Eisenkies $\left(\mathrm{Fe} \mathrm{S}_{2}\right)$. Dies zeigen die mir vorliegenden Stufen in trefflicher Weise.

Wir haben durchaus nicht nöthig uns den Vorgang verschieden vorzustellen von dem Process, der sich täglich vor unseren Augen in den

*) Erst neuerdings wurde von J. Ho ck a u ein Halotrichit aus dem Vilnösthale in Tirol beschrieben (Verhandlungen der k. k. geolog. Reichsanstalt 1887, 6, $152 \mathrm{ff}$.). Dieses Mineral ist nach der Analyse aber offenbar kein Halotrichit, sondern ein Keramohalit (Haarsalz), denn die Zusammensetzung entspricht der Formel $\left(\mathrm{Al}, \mathrm{Fe}_{2} \mathrm{O}_{3} 3 \mathrm{SO}_{3}+18 \mathrm{aq}\right.$, nicht aber dem der Zusammensetzung nach alaunäbnlichem Halotrichit ( $\mathrm{Glocker}$ ) von der Formel $\mathrm{Fe} \mathrm{Al}_{2} \mathrm{~S}_{4} \mathrm{O}_{16}+24$ aq, wo das Eisen als Oxydul vorhanden ist. 
Sammlungen*) abspielt und oft die schönsten Stufen geschwefelter Metalle oder die schönsten Versteinerungen, welche Eisenkies fuhren oder ganz aus demselben bestehen, dem Untergange weiht. Sobald der Impuls zur Umsetzung der Verbindung $\mathrm{Fe} \mathrm{S}_{2}$ in schwefelsaures Eisenoxydul gegeben ist, vollzieht sich dieselbe unabweisbar selbst in einer Atmosphare, welche nur ganz geringe Mengen Feuchtigkeit enthält, und Jedermann weiss, wie leicht sich Eisenvitriol an der Luft in schwefelsaures Eisenoxyd umsetzt. Aher selbst bei vollständiger Oxydation des Eisens erubrigt, wie man aus nachstehender Gleichung sieht, noch Schwefelsäure.

$$
2\left(\mathrm{Fe} \mathrm{S}_{2}\right)+15 \mathrm{O}=\mathrm{Fe}_{2} \mathrm{~S}_{3} \mathrm{O}_{13}+\mathrm{SO}_{3} .
$$

Die freie Schwefelsäure wirkt auf das umgebende Gestein ein und bildet mit dessen Basen Salze, wie den Halotrichit, Keramohalit, Gyps etc. Erst auf dem Wege nochmaliger Zersetzung werden im Laufe der Zeil unter dem Einflusse der eingeschlossenen oder umgebenden Gesteinsmassen aus diesen neutralen Oxydsulfaten basische Salze entstehen.

Es ist also wahrscheinlich und einleuchtend, dass auch die Sulfatlager von Tierra amarilla sich rein unterdem Einfluss der Atmosphäre undibrer Feuchtigkeit gebildet haben durch Zersetzung von Eisenkies.

Diese Verhältnisse finden einen sprechenden Beweis in der Beschaffenheit und Zusammensetzung der Stufen. Diejenigen nämlich, welche den Römerit in grosser Menge enthalten oder fast ganz aus diesem Mineral bestehen, sind stets mil mehr oder weniger Eisenvitriol vergesellschaftet und umschliessen zahlreiche Körner von Eisenkies. Auf ibrer Oberfäche oder in Drusenräumen in der Nähe derselben finden sich kleine Partien von Quenstedtit. - Andere Stufen, welche vorzugsweise aus Coquimbit bestehen, sind überzogen mil Kryställchen des basischen Copiapit und enthalten in kleinen Partien eisenhaltigen Kupfervitriol und den Ke ramohalit $\left(\mathrm{Al}_{2} \mathrm{~S}_{3} \mathrm{O}_{12}+18 \mathrm{aq}\right)$. - Der Halotrichit bildet seinerseits Stufen, welche entsprechend seinem Eisenoxydulgehalt stets mit Römerit, selten mit etwas Coquimbit associirt sind. - Auch der Stypticit bildet grössere selbständige Massen, welche nur durch ziemlich unbedeutende Mengen eines gelblicben Quarzsandes mit vielen kleinen Gypskryställehen und dem unter 11. erwäbnten noch nicht untersuchten Mineral verunreinigt sind.

*) In der That hatte ich auch Gelegenheit, bei den Zerselzungsproducten eines Eisenkies ohne näheren Fundort in der hiesigen geologischen Landessammlung neben Eisenvitriol und einem brăunlichen Sulfat, das wahrscheinlich Römerit sein dürfte, kleine, mikroskopische gelbe Blattchen zu beobachten, welche nach Form und optischen Eigenschaften dem Copiapit vollständig entsprechen. Sie bilden einen leichten Ueberzug auf dem noch nicht oxydirten Sulfat. 